The Astrophysical Journal Supplement Series, 90:713-717, 1994 February

(c) 1994. The American Astronomical Socicty. All rights reserved. Printed in U.S.A.

\title{
EFFICIENCY FOR ELECTRON ACCELERATION IN SOLAR ENERGY RELEASE REGION AS ESTIMATED IN THE CONTEXT OF PLASMA MECHANISM OF RADIO EMISSION
}

\author{
B. N. LeVin, V. M. Fridman, ANd O. A. Sheiner \\ Radiophysical Research Institute (NIRFI), B. Pecherskaya (Lydov.) str., 2S/14, 603600, Nizhny Novgorod, Russia \\ Received 1993 March 1; accepted 1993 August 17
}

\begin{abstract}
The paper is devoted to the interpretation of spectrographic data within the frequency range $8-12 \mathrm{GHz}$. Some spectral characteristics of weak solar steplike radio bursts are reported. To explain some parameters of the emission the ability to keep superthermal electrons in a magnetic trap with dense inhomogeneous plasma is theoretically studied. On the basis of this model we estimate the energy and number of accelerated electrons, and the flux density of precipitating electrons which are the source of enhanced plasma radiation. For the obtained efficiency of electron acceleration the calculated intensity of gyrosynchrotron emission proves to be less than the observed one.
\end{abstract}

Subject headings: acceleration of particles — radiation mechanisms: nonthermal — Sun: flares -

Sun: radio radiation

\section{INTRODUCTION}

An efficiency of acceleration of superthermal particles in energy release region is often estimated on the basis of their radio emission. Quantitative estimation of emitting particles depends on proper choosing of the mechanism of generation for the emission. The minimal portion of superthermal particles corresponds to the model of the most efficient generation.

In order to interpret the microwave radio events, the gyrosynchrotron radiation model is usually used. We carry out the comparative analysis of efficiencies of generation in frames of gyrosynchrotron mechanism and plasma one for the case of one and the same radio emission. This study shows that plasma mechanism of emission (radio radiation by Langmuir wave turbulence) can be more efficient just at so high a frequency as $f=10 \mathrm{GHz}$. It leads to estimations of quantity and typical energy of accelerated electrons different from those in case of gyrosynchrotron interpretations.

\section{STEPLIKE BURSTS}

We analyzed our observations of weak radio bursts made using the sweeping spectrograph-polarimeter in $8-12 \mathrm{GHz}$ frequency range (Panfilov \& Tikhomirov 1981; Lebedev et al. 1985). Weak bursts seem to be the signature of the single most efficient mechanism of generation, while for strong events the contributions of several emission processes are more probable.

During the two months of observation in 1981 November and 1983 October the radio telescope RT-22 of Crimea Astrophysical Observatory was used to receive records of 18 weak ( the radio flux was less than 10 s.f.u.) broad-band events. Their time profiles are like a step having relatively sharp growth (typical scale $t \sim 3 \mathrm{~s})$ and slow decay $(t \sim 100 \mathrm{~s})$ (Fig. 1).

Steplike bursts observed have some interesting properties. There are two of all bursts where the impulsive narrow-band drifting components have been found during the first stage of emission. This kind of emission has the bandwidth $\Delta f \sim 2-4$ $\mathrm{GHz}$, the life time 3-5 s, and the positive frequency drift $\sim 2$ $\mathrm{GHz} \mathrm{s}^{-1}$. The similar events named "blips" are often observed in decimeter wavelength range (Benz, Bernold, \& Dennis 1983 ) and more rare at our frequencies (Stahli \& Benz 1987; Levin, Fridman, \& Sheiner 1989; Benz, Magun, \& Shu 1991).

There is decay time frequency dependence for above mentioned steplike bursts (Fig. 2). The bursts have the negative spectral index over the frequency range observed. The degree of burst circular polarization $(p<10 \%)$ is low compared to that for the narrow-band component $(p \sim 20 \%)$.

\section{INTERPRETATION (OUTLINE OF THE MODEL)}

\subsection{Narrow-Band (nb) Component}

Interpretation (Levin \& Mel'nikov 1990, 1993) of the nbcomponent is developed on the basis of a thermal front emission model: Brown, Melrose, \& Spicer ( 1979), Vlahos \& Papadopoulos (1979), Smith \& Lillequist (1979). Common structure of the source of emission is schematically drawn in Figure 3.

The model has the following two typical features (Levin \& Mel'nikov 1990, 1993).

The plasma turbulence region proves to be strongly pressed to the thermal front. Due to this reason,

$$
\Delta f \sim f \frac{L_{\perp}}{2 L_{n_{\perp}}},
$$

where $L_{n}=n_{0}\left(\partial n_{0} / \partial r\right)^{-1}$ is the background plasma inhomogeneity scale and $L_{\perp}$ is the cross section scale of energy release region. Thus, one can observe narrow-band emission from sources for which $L_{1} \leq L_{n_{\perp}}$.

Moreover, the plasma turbulence region moves slowly together with the thermal front along the loop giving the frequency drift of emission

$$
\frac{\partial f}{\partial t} \sim f L_{n_{1}}^{-1} V_{\mathrm{fr}} / 2
$$

where $V_{\mathrm{fr}}$ is the velocity of thermal front movement. 


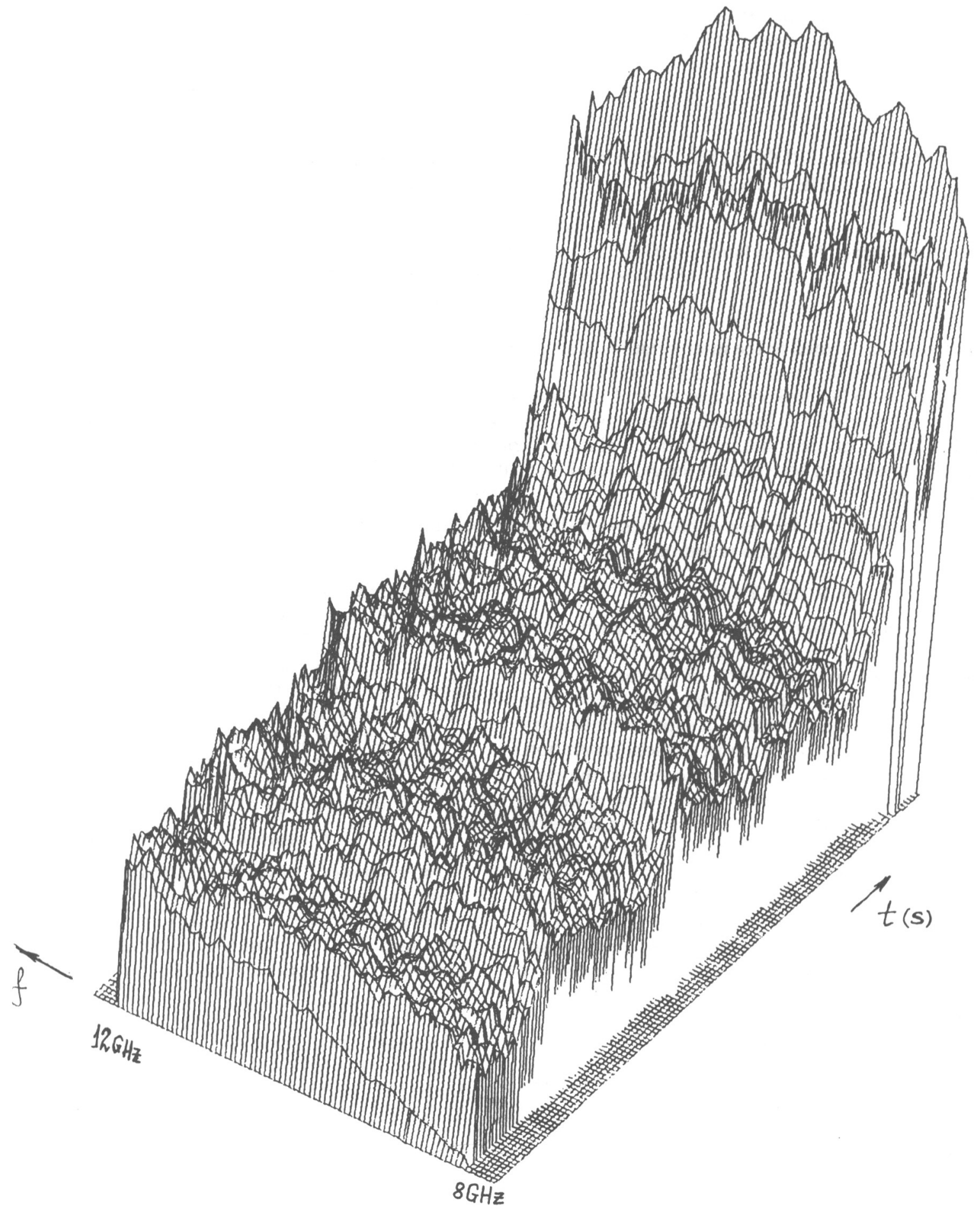

FIG. 1.-Example of perspective imaging the steplike changes in the microwave $(8-12 \mathrm{GHz})$ emission. These events are observed before the flash phase of the main flare (1981 November 14). Each check on the $t$-axis is one second. 


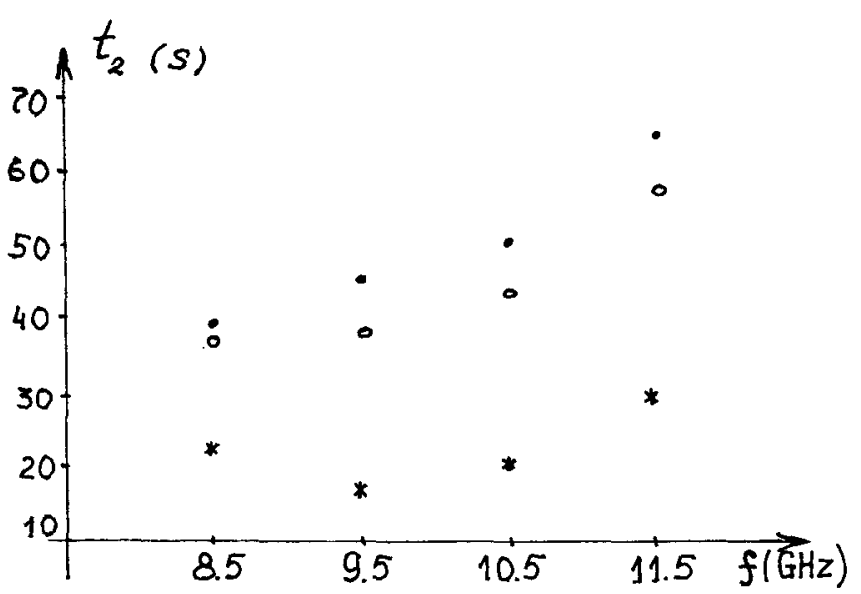

Fig. 2.-Examples of the decay time dependencies on frequency of emission for steplike bursts.

Using the observed bandwidth and frequency drift rate of the nb-component one can analyze the spatial structure of the source. For example, results of that analysis (Levin et al. 1989) are as follows: $L_{n \|} \sim 3 \times 10^{8} \mathrm{~cm}, L_{\perp} \leq 10^{8} \mathrm{~cm}$.

\subsection{Steplike Continuum (cont) Emission}

We shall provide the comparative study of two models of emission based on gyrosynchrotron and plasma mechanisms of generation. For both models the source of emission is superthermal electrons trapped by a loop of coronal magnetic field. The emitting electrons are accelerated during the first (short, $t_{1} \sim 1-3 \mathrm{~s}$ ) stage of an event. The decay time of emission $\left(t_{2} \sim 10^{2} \mathrm{~s}\right)$ is defined by the lifetime of superthermal electrons.

For the "gyrosynchrotron model" emission is yielded through the gyrosynchrotron radiation of the mildly relativistic electrons. The source of emission is localized at " $o$ "-region (top of the loop). Steplike (continuum) emission appears independently on the nb-component which is of plasma origin.

In "plasma model" the plasma turbulence is the source of emission. Therefore the emission frequency is strongly connected with the source location ("p"-region) (Fig. 3). The model explains the consecutive appearance of the narrowband and then steplike sources in visible region.

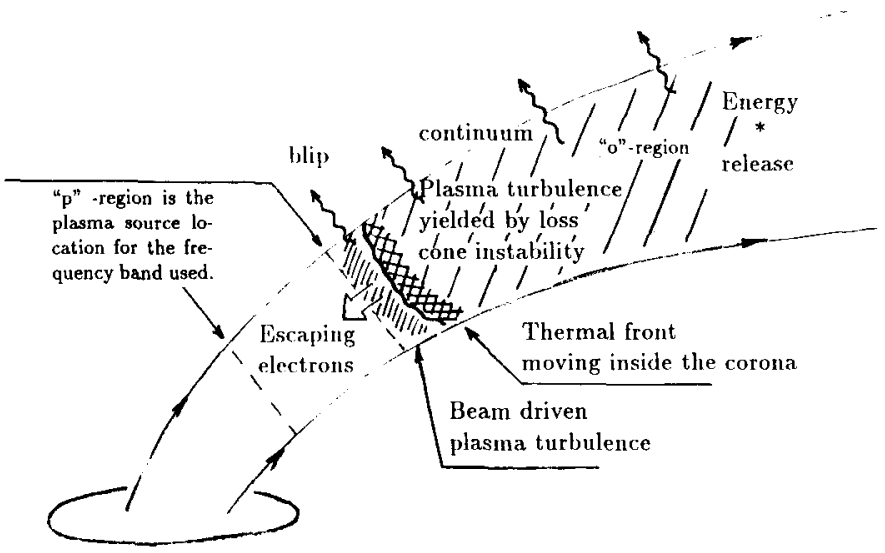

FIG. 3.- Schematic representation of the assumed source model in a coronal magnetic loop.

\subsection{Energy of Emitting Electrons}

The lifetime of the trapped electrons $\tau_{e}$ is defined by energy losses due to the collisions with background plasma (Bai 1982):

$$
\frac{\langle\Delta E\rangle}{E} \approx \frac{\gamma_{o}+1}{\gamma_{o}} \frac{16 \pi e^{4}}{m^{2} v_{s}^{4}} S_{b} \ln \Lambda \int_{0}^{s_{m}} n_{e}(s) d s .
$$

Here $\gamma_{o}$ is Lorentz-factor, $m$ is the mass, $e$ is the charge and $v_{s}$ is the velocity of the electron, $\ln \Lambda$ is the Coulomb logarithm, $s$ is the coordinate along the loop axis, $s_{m}$ is the mirroring point coordinate of an electron, and $S_{b}=\tau_{e} v_{s} / 4 s_{m}$ is the number of bounce oscillations for an electron during its lifetime.

Equation (3) takes the mean energy of the bulk of emitting electrons $E_{s}=m v_{s}^{2} / 2$ trapped in a magnetic loop in accordance with their lifetime and hence with the decay time of emission observed:

$$
\begin{aligned}
\tau_{e}^{-1} \sim-\frac{\gamma_{o}+1}{\gamma_{o}^{2}} \frac{16 \pi^{3} f_{p}^{4} \ln \Lambda}{n_{o} v_{s}^{3}} & \\
& \times \frac{L_{n}}{L_{\mathbf{H}}} \frac{\mu_{o}^{2}}{\left(1-\mu_{o}^{2}\right)^{L_{n} / L_{\mathrm{H}}} \ln \left(1-\mu_{o}^{2}\right)},
\end{aligned}
$$

where $\mu_{o}$ is the pitch angle cosine for electrons in the top of the loop.

For gyrosynchrotron model it means that distribution of fast electrons has low-energy cutoff $E<E_{s}$ and falls down sharply toward the higher energies. The spectral low of the distribution is not of interest for crude estimation of intensity of radio emission.

For the plasma model another situation is realized. The source of emission is localized in strongly divergent magnetic loop with inhomogeneous background plasma. Since the radio emission is generated in a dense plasma region the energy of emitting particles has to be great enough for their possibility to penetrate through the dense plasma up to a corresponding level. Due to this reason there is the dependence $E_{s}=E_{s}(f)$ for the plasma mechanism.

Moreover, let us mention that for the plasma model case of direct connection between the local plasma frequency and frequency of emission, equation (4) indicates the possibility of frequency dependence of the steplike burst duration observed (Fig. 2). For such dependence to appear in so narrow a frequency range for gyrosynchrotron emission seems to be less probable.

\section{GYROSYNCHROTRON EMISSION}

We assume both components (narrow-band and steplike continuum) are generating in one and the same magnetic loop. The spectral index of the continuum emission shows that it can be optically thin equilibrium emission. To make estimations let us consider the two cases for the gyrofrequencyplasma frequency ratio $a=1.5 f_{\mathrm{H}} / f_{p}$ in the source region: $a=3$ (a tenuous plasma) and $a=0.5$ (a dense plasma). Gyrosynchrotron absorption coefficient $\mu_{i}^{x, 0}$ and emissivity $a_{\omega}$ are estimated using calculations of Ramaty (1969) and Klein (1987). Free-free absorption coefficient is (Zheleznyakov 1977)

$$
\mu_{\mathrm{ff}}=\frac{f_{p}^{2} \nu_{\mathrm{eff}}^{e i}}{f^{2} c \sqrt{1-f_{p}^{2} / f^{2}}}
$$


TABLE 1

Parameters of THE Model of Gyrosynchrotron EMisSion

\begin{tabular}{|c|c|c|c|c|}
\hline Loop Model & "p"-Region & $" o "$-Region & Source in Dense Plasma & Source in Tenuous Plasma \\
\hline $\begin{aligned} \text { Model A: } & \\
$\[ \begin{array}{l}\sigma \\
=\end{array} \]$ & =1 \\
T_{0} & \sim 10^{6} \mathrm{~K}\end{aligned}$ & $\begin{array}{l}\text { " } p \text { "-region is localized } \\
\text { at top of loop }\end{array}$ & $\begin{aligned} n_{o} & \sim 3 \times 10^{11} \mathrm{~cm}^{-3}\left(f_{p} \sim 5 \mathrm{GHz}\right) \\
L_{\perp} & \sim 10^{8} \mathrm{~cm}, L_{n_{11}} \sim 3 \times 10^{8} \mathrm{~cm} \\
\rightarrow V & =L_{\perp}^{2} \times L_{n_{1}} \sim 3 \times 10^{24} \mathrm{~cm}^{3} \\
& \tau_{e} \sim 10^{2} \mathrm{~s} \rightarrow E_{s} \sim 10 \mathrm{MeV}\end{aligned}$ & $\begin{array}{l}H \sim 3 \times 10^{3} \mathrm{G} \\
\text { Low-probable case }\end{array}$ & $\begin{array}{l}H \sim 600 \mathrm{G} \\
n_{s} \sim 10^{7}-10^{8} \mathrm{~cm}^{-3} \\
N_{s}=n_{s} V \sim 3 \times 10^{31}-3 \times 10^{32}\end{array}$ \\
\hline $\begin{array}{l}\text { Model B: } \\
\qquad \begin{aligned} L_{\mathrm{H}} & \sim L_{n_{\|}} \\
\sigma & =10^{6} \\
T_{0} & \sim 10^{6} \mathrm{~K}\end{aligned}\end{array}$ & $\begin{aligned} n_{p} & \sim 3 \times 10^{11} \mathrm{~cm}^{-3} \\
\left(f_{p}\right. & \sim 5 \mathrm{GHz}) \\
L_{\perp} & \sim 10^{8} \mathrm{~cm} \\
L_{n_{1}} & \sim 3 \times 10^{8} \mathrm{~cm}\end{aligned}$ & $\begin{aligned} n_{o} & \sim 3 \times 10^{10} \mathrm{~cm}^{-3} \\
L_{\perp} & \sim 3 \times 10^{8} \mathrm{~cm}, L_{n_{\|}} \sim 10^{9} \mathrm{~cm} \\
\rightarrow V & \sim 10^{26} \mathrm{~cm}^{3} \\
\tau_{e} & \sim 10^{2} \mathrm{~s} \rightarrow E_{s} \sim 1 \mathrm{MeV}\end{aligned}$ & $\begin{array}{l}H \sim 10^{3} \mathrm{G} \\
n_{s} \sim 5 \times 10^{2}-5 \times 10^{3} \mathrm{~cm}^{-3} \\
N_{s} \sim 5 \times 10^{28}-5 \times 10^{29}\end{array}$ & $\begin{array}{l}H \sim 200 \mathrm{G} \\
n_{s} \sim 3 \times 10^{4}-3 \times 10^{5} \mathrm{~cm}^{-3} \\
N_{s} \sim 3 \times 10^{30}-3 \times 10^{31}\end{array}$ \\
\hline $\begin{array}{l}\text { Model C: } \\
\qquad \begin{aligned} L_{\mathbf{H}} & \sim L_{n_{\|}} \\
\sigma & =30^{6} \\
T_{0} & \sim 10^{6} \mathrm{~K}\end{aligned}\end{array}$ & $\begin{aligned} n_{p} & \sim 3 \times 10^{11} \mathrm{~cm}^{-3} \\
\left(f_{p}\right. & \sim 5 \mathrm{GHz}) \\
L_{\perp} & \sim 10^{8} \mathrm{~cm} \\
L_{n_{4}} & \sim 3 \times 10^{8} \mathrm{~cm}\end{aligned}$ & $\begin{aligned} n_{o} & \sim 10^{10} \mathrm{~cm}^{-3} \\
L_{\perp} & \sim 6 \times 10^{8} \mathrm{~cm}, L_{n_{1}} \sim 2 \times 10^{9} \mathrm{~cm} \\
\rightarrow V & \sim 7 \times 10^{26} \mathrm{~cm}^{3} \\
\tau_{e} & \sim 10^{2} \mathrm{~s} \rightarrow E_{s} \sim 0.5 \mathrm{MeV}\end{aligned}$ & $\begin{array}{l}H \sim 600 \mathrm{G} \\
n_{s} \sim 2 \times 10^{2}-2 \times 10^{3} \mathrm{~cm}^{-3} \\
N_{s} \sim 10^{29}-10^{30}\end{array}$ & $\begin{array}{l}H \sim 100 \mathrm{G} \\
n_{s} \sim 10^{4}-10^{5} \mathrm{~cm}^{-3} \\
N_{s} \sim 7 \times 10^{30}-7 \times 10^{31}\end{array}$ \\
\hline
\end{tabular}

Taking into account the Rayleigh-Jeans formula

$$
S_{f}=\frac{a_{\omega}\left(n_{s}\right)}{\max \left\{\mu_{\mathrm{ff}}, \mu_{i}^{x, 0}\right\}} \frac{\Delta \Omega}{2 \pi},
$$

we obtain the concentration of emitting electrons $n_{s}$ for the level of emission $S_{f}=1-10$ s.f.u. Here $\Delta \Omega$ is the source angular dimension.

Thus, spatial scales near the bottom of the loop we estimate using a nb-component analysis. Then assuming the value of loop divergence parameter $\sigma$ we obtain the scales of the top of the loop. On the base of the formulae mentioned above we can crudely estimate the main parameters of the source of gyrosynchrotron emission (Table 1).

\section{PLASMA TURBULENCE RADIO EMISSION}

\subsection{Enhanced Plasma (Langmuir) Noise}

In the presence of superthermal electrons there are enhanced fluctuations of Langmuir waves in background plasma (Perkins \& Salpeter 1965; Tidman \& Dupree 1965). For twoMaxwellian ( $T_{0}$ and $T_{s}$ ) plasma the spectral density of Langmuir waves in the equilibrium state can be estimated from the transfer equation (Levin 1982, 1987).

For more dense populations of superthermal electrons their distribution can become unstable, for example, due to the loss cone. The marginal condition for loss-cone instability $\gamma \sim d$, where the increment of instability (Melrose 1977, 1980) is $\gamma \sim$ $0.2 \pi f_{p} n_{s} / n_{0}$, plasma wave decrement is $d \sim \nu_{\text {eff }}^{e i} / 2$.

Some results of estimations of the equilibrium plasma turbulence level $T_{\mathrm{pl}}$ and concentration of the superthermal electrons in the marginal condition for loss-cone instability are given in Figure 4.

\subsection{Conversion Processes to the Radio Radiation}

It is well-known that radio radiation from enhanced plasma turbulence is possible due to Rayleigh scattering of plasma waves $\left(f \sim f_{p}\right)$; or / and due to coalescence of two plasma waves $\left(f \sim 2 f_{p}\right)$; or/and due to coalescence of Langmuir waves and plasma waves of any low-frequency branches $\left(f \sim f_{p}\right)$ (Melrose 1980 ).

There is a strong collisional depression of plasma radio emission outside of source (escaping problem) $S_{f}^{\text {obs }}=$ $\exp \left\{-\tau_{\mathrm{ff}}^{t}\right\} S_{f}^{\text {sour }}$ (Melrose 1980), where $\tau_{\mathrm{ff}}^{t}=\int \mu_{\mathrm{ff}} d l$ is the optical depth of emission. For the second harmonic of local plasma frequency $\left(f \sim 2 f_{p}\right.$ ) using equation ( 5 ) one can obtain $\tau_{\text {ff }}^{t} \sim 10^{-7} L_{n}$; for the fundamental plasma emission $\left(f \sim f_{p}\right) \tau_{\mathrm{ff}}^{t} \sim 6 \times 10^{-6} L_{n}$.

Bearing in mind the estimations of Figure 4 and the probable scales $L_{n} \sim 10^{7}-10^{9} \mathrm{~cm}$ we come to the following conclusions. First, the emission is possible on the second harmonic only in the direction of sharp fall down of background plasma concentration, i.e., transverse to the loop. In our case it is to be the limb emission. Second, the largest optical depth for coalescence of the equilibrium plasma waves is estimated as being too small $\left(\sim 10^{-5}\right)$ to explain the steplike emission observed. This is the reason to consider the coherent emission only $\left(n_{s} \geq 10^{6}\right)$.

\subsection{Fokker-Planck Equation for Electrons in Inhomogeneous Magnetic Trap}

The estimated concentration of emitting electrons corresponds to the source region (" $p$ "-region). However, their lifetime in the $p$-region is too small due to the high plasma density there. We can explain the observed burst duration in the context of the plasma mechanism of radiation assuming these electrons to be precipitated ones.

According to MacDonald \& Walt (1961), Krinberg (1978, p. 172), and Lu \& Petrosyan (1988) the superthermal electron behavior in the magnetic loop is described by the following equation:

$$
\frac{\partial F}{\partial t}=\frac{c}{\lambda_{o} \beta^{3}} \frac{1}{\mu_{0} S_{\mu}} \frac{\partial}{\partial \mu_{0}}\left[A_{\mu} \frac{1-\mu_{0}^{2}}{\mu_{0}} \frac{\partial F}{\partial \mu_{0}}\right]+\frac{c}{\lambda_{o} \beta^{2}}\left\langle n_{c}\right\rangle \frac{\partial F}{\partial \beta} .
$$

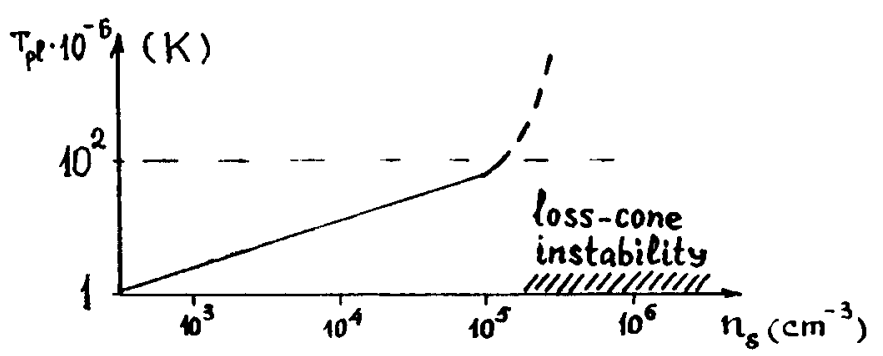

FIG. 4.-Estimation of the level of the equilibrium plasma noises against the density of population of superthermal electrons. 
Here $F=F\left(v, \mu_{0}, t, s\right)$ is the electron distribution function, $\lambda=$ $10^{24} / \ln \Lambda$,

$$
\begin{gathered}
S_{\mu}=\int_{0}^{s_{m}} d s / \mu\left(s, \mu_{0}\right), \\
A_{\mu}\left(\mu_{0}\right)=\int_{0}^{s_{m}} d s n_{e} \mu\left(s, \mu_{0}\right) H_{0}^{2} / H^{2}(s), \\
\left\langle n_{e}\right\rangle=\frac{1}{S_{\mu}} \int_{0}^{s_{m}} d s n_{e}(s) / \mu\left(s, \mu_{0}\right), \quad \beta=v / \mathcal{c} .
\end{gathered}
$$

For exponential inhomogeneous media with scales $L_{\mathrm{H}}$ and $L_{n_{1}}$ and for the stationary case $t \ll 10^{2} \mathrm{~s}$ one can obtain the equation for $n_{s}\left(\mu_{0}\right)=\int F v^{2} d v$ :

$$
\frac{1}{\mu_{0} S_{\mu}} \frac{d}{d \mu_{0}}\left[A_{\mu} \frac{1-\mu_{0}^{2}}{\mu_{0}} \frac{d n_{s}\left(\mu_{0}\right)}{d \mu_{0}}\right]-3\left\langle n_{e}\right\rangle n_{s}\left(\mu_{0}\right)=0,
$$

with boundary condition $n_{s}\left(\mu_{0}\right)=n_{s}\left(\mu_{p}\right) \sim n_{s} / 2 \pi$. As the $n_{s}$ value we will take the estimation of the previous subsection $n_{s} \sim 10^{6} \mathrm{~cm}^{-3}$.

This equation can be solved numerically for different $L_{\mathrm{H}} / L_{n}$ ratios (Fig. 5). Now using the same way as for the gyrosynchrotron model we can crudely calculate the number of accelerated electrons in the possible plasma model of the source of emission (Table 2).

For a power-law energy spectrum of superthermal electrons with index $\delta \sim 3.5-4$ the density of mildly relativistic electrons $\left(E_{s} \sim 0.5 \mathrm{MeV}\right)$ in this loop is on order $n_{s} \sim 10^{3} \mathrm{~cm}^{-3}$, i.e., less than one needs in the gyrosynchrotron case.

\section{CONCLUSIONS}

The plasma mechanism of radiation can be considered as an alternative one for solar microwave radio emission at least up

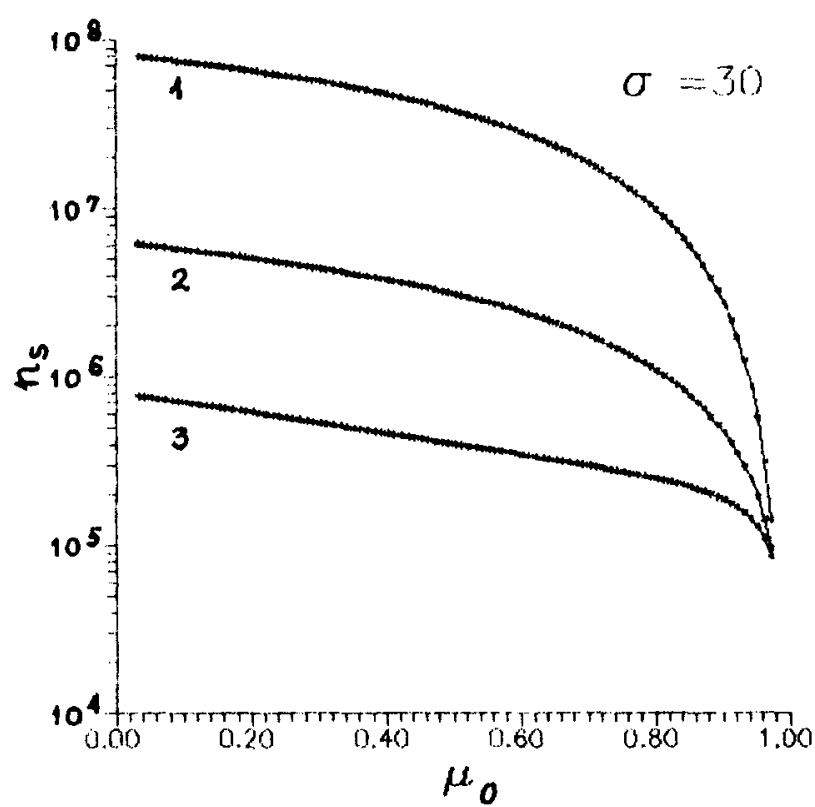

FiG. 5.-Calculated angular part of the distribution function of trapped electrons $n_{s}\left(\mu_{0}\right)=\int F v^{2} d v$ in the top of the loop for different values $L_{\mathbf{H}} / L_{n_{\mathbf{I}}}$. Here $1-L_{\mathbf{H}} / L_{n_{\mathbf{I}}}=3 ; 2-L_{\mathbf{H}} / L_{n_{\mathbf{I}}} \ll 1 ; 3-L_{\mathbf{H}} / L_{n_{\mathbf{I}}}=2$.

to a frequency range of $10 \mathrm{GHz}$. In frames of this mechanism the weak steplike continuum can be radiated by Langmuir turbulence at the dense plasma. Plasma turbulence is generated by a small precipitating portion of the superthermal electrons surviving during the long time in the top of the coronal magnetic loop. According the our estimations the flux density of precipitating particles of energy $E_{s} \sim 30 \mathrm{keV}$ is $J_{s} \sim 10^{15}$ $\mathrm{cm}^{-2} \mathrm{~s}^{-1}$.

TABLE 2

Parameters of the Model for Radio Radiation of Plasma Turbulence

\begin{tabular}{llcc}
\hline \hline Loop Model & "p"-Region & "o"-Region & Source of Emission \\
\hline Model D: & $n_{p} \sim 3 \times 10^{11} \mathrm{~cm}^{-3}$ & $n_{o} \sim 3 \times 10^{8} \mathrm{~cm}^{-3}$ & \\
$L_{\mathrm{H}} \sim 2 L_{n_{\|}}$ & $\left(f_{p} \sim 5 \mathrm{GHz}\right)$ & $L_{\perp} \sim 5 \times 10^{8} \mathrm{~cm}_{1} L_{n_{1}} \sim 2 \times 10^{9} \mathrm{~cm}$ & $n_{s} \sim 3 \times 10^{7} \mathrm{~cm}^{-3}$ \\
$\sigma=30$ & $L_{\perp} \sim 10^{8} \mathrm{~cm}$ & $\rightarrow V \sim 5 \times 10^{26} \mathrm{~cm}^{3}$ & $N_{s} \sim 10^{34}$ electrons \\
$T_{0} \sim 10^{6} \mathrm{~K}$ & $L_{n_{\|}} \sim 3 \times 10^{8} \mathrm{~cm}$ & $\tau_{e} \sim 10^{2} \mathrm{~s} \rightarrow E_{s} \sim 30 \mathrm{keV}$ & $\cdots$ \\
\hline
\end{tabular}

\section{REFERENCES}

Bai, T. 1982, ApJ, 259, 341

Benz, A. O., Bernold, T. E. X., \& Dennis, B. R. 1983, ApJ, 271, 355

Benz, A. O., Magun, A., \& Su, H. 1991, in Abstracts of 4th CESRA Workshop, ed. L. Vlahos (Ouranoupolis: CESRA), 9

Brown, J. C., Melrose, D. B., \& Spicer, D. S. 1979, ApJ, 228, 592

Klein, K.-L. 1987, A\&A, 183, 341

Krinberg, I. A. 1978, Kinetics of Electrons in Earth Iono- and Plasmasphere (Moscow: Nauka)

Lebedev, E. I., Panfilov, Yu. D., Tikhomirov, V. A., \& Tikhomirov, Yu. V. 1985, Bull. Inventions 21, Author's Certificate 1160329, USSR

Levin, B. N. 1982, A\&A, 111, 71

1987, Izv. VUZov Radiofizika, 30, 1505

Levin, B. N., Fridman, V. M., \& Sheiner, O. A. 1989, Izv. VUZov Radiofizika, 32, 516

Levin, B. N., \& Mel'nikov, V. F. 1990, Astron. Nachr., 311, 405 . 1993, Solar Phys., in press
Lu, E. T., \& Petrosyan, V. 1988, ApJ, 327, 405

MacDonald, W. M., \& Walt, M. 1961, Ann. Phys., 15, 44

Melrose, D. B. 1977, Izv. VUZov Radiofizika, 20, 1369

— 1980 , Plasma Astrophysics, Vol. II (New York: Gordon \& Breach), 423

Panfilov, Yu. D., \& Tikhomirov, V. A. 1981, Bull. Inventions 11, Author's Certificate 815671, USSR

Perkins, F., \& Salpeter, E. E. 1965, Phys. Rev., A55, 139

Ramaty, R. 1969, ApJ, 158, 753

Smith, D. F., \& Lillequist, C. G. 1979, ApJ, 232, 582

Stahli, M., \& Benz, A. O. 1987, A\&A, 175, 271

Tidman, D. A., \& Dupree, T. H. 1965, Phys. Fluids, 8,1860

Vlahos, L., \& Papadopoulos, K. 1979, ApJ, 233, 717

Zheleznyakov, V. V. 1977, Electromagnetic Waves in Cosmic Plasma (Moscow: Nauka) 